\title{
Niveles de Resiliencia y Estado de Salud General percibido de Estudiantes Universitarios de Psicología, Víctimas del Conflicto Armado en Sucre
}

\author{
Resilience Levels and General Health Percecption in students of \\ Psychology, Victims of the Armed Conflict in Sucre
}

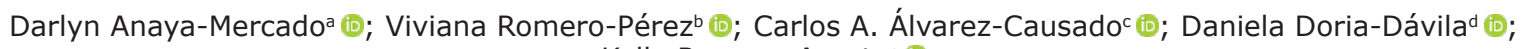
Kelly Romero-Acostae (i)

\section{RESUMEN}

El objetivo del presente trabajo de investigación es determinar los niveles de resiliencia, el estado de salud general percibido y los aspectos relacionados con los hechos victimizantes y con la atención psicosocial, de Estudiantes Universitarios Víctimas del Conflicto en Sucre. Este es un estudio descriptivo, de enfoque cuantitativo. Participaron 47 Estudiantes Víctimas del Conflicto quienes completaron los siguientes instrumentos: un instrumento de datos sociodemográficos (ad hoc), compuesto por las variables sexo, edad y nivel socioeconómico. Un instrumento (ad hoc) que indagaba sobre la cantidad y el tipo de hechos victimizantes vividos y sobre la atención psicosocial recibida. También se contó con una escala para medir la salud general percibida y una escala de resiliencia. Los resultados evidenciaron que el tipo de hecho victimizante más frecuente fue el desplazamiento forzado. Si bien, pocos Estudiantes recibieron ayuda psicológica por su experiencia vivida en el Conflicto, la gran mayoría presentó una resiliencia alta y moderada. La media del cuestionario de salud general percibida fue 6,81 (desviación estándar=1,38).

Palabras clave: Desplazamiento; Conflicto; Estudiantes; Resiliencia; Salud mental

\begin{abstract}
The objective of the present research work is to determine the levels of resilience, the general perceived state of health and the aspects related to the victimizing facts and with the psychosocial attention of University Students Victims of the Conflict in Sucre. This is a descriptive, quantitative approach study. Forty-seven Student Victims of the Conflict participated, completing the following instruments: a sociodemographic (ad hoc) data instrument, made up of the variables sex, age, and socioeconomic level. An (ad hoc) instrument that inquired about the amount and type of victimizing events experienced and about the psychosocial care received. There was also a

aFundación Semillas de Vida para Colombia - FUNSEVIDA, área de Coordinación. Sincelejo, Colombia. Dadamer16@gmail.com bFundación para el Desarrollo Comunitario - La Luz, área de Psicología. Sincelejo, Colombia. romero.viviana2803@gmail.com 'Independiente. Sincelejo, Colombia. carlos.alvarezc@cecar.edu.co

¿Corporación Universitaria del Caribe CECAR, programa de Psicología. Sincelejo, Colombia. Doria969@gmail.com

eCorporación Universitaria del Caribe CECAR, programa de Psicología. Sincelejo, Colombia. kelly.romero@cecar.edu.co

Como citar (APA)

Anaya-Mercado, D.; Romero-Pérez, V.; Álvarez-Causado, C.A.; Doria-Dávila, D.; Romero-Acosta, K. (2020). Niveles de Resiliencia y Estado de Salud General percibido de Estudiantes Universitarios de Psicología Víctimas del Conflicto Armado en Sucre. Búsqueda, v. 7, n. 24, e465. https://doi.org/10.21892/01239813.465
\end{abstract}


scale to measure perceived general health and a resilience scale. The results showed that the most frequent type of victimizing act was forced displacement. Although few students received psychological help due to their lived experience in the conflict, the vast majority presented high and moderate resilience. The mean of the general perceived health questionnaire was 6.81 (standard deviation $=1.38$ ).

Keywords: Displacement; Conflict; Students; Resilience; Mental Health

\section{INTRODUCCIÓN}

La historia de Colombia ha estado marcada por un factor que supone el riesgo para el buen estado de salud mental y física en la población. Dicho factor es el Conflicto Armado, el cual ha dejado centenares de víctimas, a través de las diferentes modalidades de violencia a las que fueron sometidas. El Desplazamiento es una consecuencia de la situación de violencia en la que se sumió el país; este genera el quebrantamiento de la estructura y los vínculos familiares, pérdida de la vivienda y la tierra, desempleo, incertidumbre de un futuro desconocido, dificultades de adaptación en los niños y adolescentes, entre otras (Ramírez-Giraldo, Hernández-Bustamante, Romero-Acosta, \& Porras-Mendoza, 2017).

Según el Centro Nacional de Memoria Histórica (CNMH), el total de víctimas fatales, entre 1958 y 2018, fueron 261.619, de las cuales, 214.584 eran víctimas civiles; 46.675, combatientes; y 360 , personas de las cuales no se reporta información (Centro Nacional de Memoria Histórica, 2018). Del mismo modo, el Departamento de Sucre representa una de las regiones que más ha sido golpeada por esta problemática, debido a la alta cifra de personas que han sido reconocidas como víctimas en la región. De acuerdo con la Red Nacional de Información (2018), la cifra asciende a 301.686, según el corte del 01 de mayo de 2018. Las cifras también indican que en la subregión de Los Montes de María han sucedido alrededor de 18 masacres; resaltando, entre ellas, la de El Salado, Chengue y Macayepo.

Estas masacres llevaron a una migración masiva de personas hacia los cascos urbanos, principalmente a la capital del Departamento, Sincelejo (Centro Nacional de Memoria Histórica, 2018). En consecuencia de ello, en el año 2011, el Congreso de Colombia crea la Ley 1448, la cual tiene como objetivo establecer un conjunto de medidas judiciales, administrativas, sociales y económicas, individuales y colectivas, para las víctimas de las infracciones al Derecho Internacional Humanitario, y normas internacionales de Derechos Humanos ocurridas con ocasión del Conflicto Interno (Congreso de Colombia, 2011).

Entre los hechos que vulneraron los derechos humanos de los sucreños, se encuentran: la violencia sexual, las masacres, las desapariciones forzadas, el desplazamiento forzado, las torturas, y un gran número de acciones humillantes y/o de amenazas que obligaron a muchas víctimas a marcharse de sus tierras (Juarez \& Guerra, 2011). Herrera (2016) señala que las personas que hacen parte y que vivieron el Conflicto Armado, desencadenan una gran cantidad de afectaciones psicológicas, hecho que confirma un estudio realizado por Médicos Sin Fronteras (2013), el cual halló que el $34 \%$ de la población víctima en Cauca, Caquetá y Putumayo presentaba trastorno de ansiedad y depresión, pues manifestaban desesperanza, ideación suicida e incluso miedo de volver a sus tierras por la situación que tuvieron que vivir. Castaño y sus colaboradores (2018), realizaron una investigación sobre la salud mental en personas víctimas del Desplazamiento por la violencia en Colombia en tres partes del país - Buenaventura, Medellín y Bogotá-, en las cuales identificaron que, en relación con la salud mental de las personas, los trastornos más predominantes en la población fueron: la fobia específica, el estrés postraumático y la depresión mayor.

En lo que respecta a Sucre, Castillo et al., (2017) hallaron que los adultos mayores Víctimas del Conflicto presentan depresión y, también, Ramírez et al., (2017) hallaron altos porcentajes de depresión mayor en las poblaciones de Chengue y de Libertad, tiempo después de las masacres y de la vulneración de los derechos humanos. Ramirez et al., (2017) hallaron que la población de Chengue tuvo un porcentaje más alto de personas con depresión en comparación con Libertad; 
y señalan que una de las razones puede ser la pérdida del tejido social de los habitantes de Chengue, junto con la vivencia del hecho traumático, esto es, la masacre. Es decir, que contar con una red social puede atenuar las vivencias traumáticas de una masacre; la familia y los amigos pueden ayudar a afrontar esta terrible situación.

En efecto, existen diferentes mecanismos para afrontar situaciones de estrés; de hecho, algunas personas son capaces de enfrentar las situaciones adversas y aprender de ellas. A este fenómeno se le llama resiliencia. Muñoz (2019) define la resiliencia como la capacidad que tienen los seres humanos para enfrentar la adversidad y lograr sobrellevar las tragedias, los traumas, o el estrés severo, ocurridos en este caso por parte de grupos armados. A partir de esto, las victimas logran identificar las dificultades y, así, visionarse y reconstruir su proyecto de vida. Esto se demuestra, también, en el estudio realizado por Hewitt y sus colegas (2016) con Adultos Víctimas del Conflicto Armado, en la que se encontró que $61 \%$ de los participantes, presentó un nivel moderado, y el $32 \%$ un nivel alto de resiliencia.

Otro aspecto sobresaliente sobre los estudios de resiliencia es lo expuesto por Bonilla (2013), quien postula que el núcleo familiar juega un papel importante en el proceso de obtención de recursos internos, que beneficien la conducta resiliente que desarrollan las personas, después de vivir un acontecimiento traumático como el Desplazamiento Forzado. El poder tener a alguien cercano y expresar sus pensamientos y sentimientos produce un efecto de confianza. Es por esto que las familias resilientes se caracterizan por tener la capacidad de enfrentar cambios y de seguir unidos a pesar de la adversidad. Entonces, las familias resilientes pueden favorecer a los Jóvenes Víctimas del Conflicto Armado.

También, la sociedad puede favorecer a las jóvenes víctimas. Algunos elementos de protección socio-comunitaria que ayudan a tener una capacidad de resiliencia en los jóvenes son: el ingreso a organizaciones o fundaciones que tengan como objetivo velar por los derechos humanos y el bienestar social; el ingreso a grupos deportivos o grupos comunitarios; el ingreso a los estudios universitarios; entre otros. Estos elementos benefician el desarrollo de un comportamiento resiliente verdadero. Así, las instituciones de educación superior también pueden apoyar a los jóvenes en su proceso de resiliencia y en su adecuada adaptación a la función social.

Hoy por hoy, las Universidades colombianas albergan en sus aulas a Grupos de Estudiantes Víctimas del Conflicto, que, posiblemente, tienen secuelas psíquicas de la violencia vivida en sus lugares de origen. Esta cifra aumenta en tanto el Instituto Colombiano de Crédito Educativo y Estudios Técnicos en el Exterior-Icetex, ha brindado en los últimos años planes de becas dirigidos a incorporar a Jóvenes Víctimas del Conflicto a las aulas de clase universitarias. En las Universidades de Sucre muchos Estudiantes, beneficiados por el plan de crédito del Icetex, son Víctimas del Conflicto Armado. No obstante, ¿se tiene información sobre la experiencia vivida por estos Estudiantes en el Conflicto?, ¿se sabe cuál es su nivel de resiliencia o si han recibido o requieren apoyo psicológico? La literatura científica nos indica que vivir situaciones traumáticas puede causar una elevación de angustia en cualquier tipo de persona e, incluso, para aquellas que no sufren en ese momento algún trastorno mental. Por esto, es conveniente actuar rápidamente en el proceso de apoyo (Hewitt Ramírez, y otros, 2016). Si las Universidades no saben el tipo de afectación que tienen sus Estudiantes a causa del Conflicto, no podrán brindar un apoyo eficiente cuando sea necesario. En este sentido es útil conocer las vivencias del Conflicto Armado que tienen los Estudiantes Universitarios, para que, así, las Universidades, como en la que se llevó a cabo este estudio, puedan dar apoyo al proceso académico y a la construcción del proyecto de vida de estos jóvenes, de manera que se contribuya al mejoramiento de la calidad de vida de los mismos (Rodríguez, De La Torre, \& Miranda, 2002).

El gremio de los Estudiantes de Psicología, es uno de los más numerosos de la Universidad, tanto pública como privada. Los Psicólogos son aquellas personas que pueden ayudar a otros en situaciones de estrés. Probablemente, los Psicólogos en formación que actualmente son Víctimas del Conflicto, serán los que atiendan a las Víctimas del Conflicto una vez terminen sus estudios. Si estos Estudiantes, ya marcados por el Conflicto, logran tomar su experiencia como una fortaleza, podrán intervenir de mejor manera a las Víctimas del Conflicto Armado y podrán ofrecer un servicio terapéutico de calidad. 
De igual forma, la Ley 1616 de 2013, en el Artículo 18, estipula que los equipos interdisciplinarios deben contar con un grupo de profesionales, entre ellos los del servicio de Psicología, para que garanticen el respeto, la dignidad y los derechos humanos. Esto quiere decir, que los profesionales de Psicología que atienden a población vulnerable deben estar preparados para brindar una atención adecuada, la cual será mucho más idónea si cuentan con los recursos psicológicos para hacerlo. En este sentido, el estado de salud mental y los niveles de resiliencia del personal de la salud, son cruciales para brindar una mejor atención psicosocial a quienes lo necesiten.

Por lo que se refiere a la salud mental, no se tiene información sobre esto, tampoco sobre los niveles de resiliencia de Víctimas del Conflicto Armado en la Universidad, ni sobre las características de la vivencia del Conflicto, tales como tipo de hecho victimizante; número de hechos victimizantes vividos; percepción de la influencia de las vivencias traumáticas en el proceso de aprendizaje, en su elección de carrera o en su vida familiar. Tampoco se ha indagado sobre el papel de la Universidad frente a todas estas vivencias, sobre todo en la atención psicosocial. Por todo lo anterior, el objetivo de este estudio es determinar los niveles de resiliencia, el estado de salud mental percibido, y los aspectos relacionados con los hechos victimizantes y con la atención psicosocial, de Estudiantes Universitarios Víctimas del Conflicto Armado en Sucre.

\section{MÉTODO}

Paradigma y Enfoque. La presente investigación es de paradigma positivista, de enfoque cuantitativo, y de tipo descriptivo (Sampieri, Collado, \& Lucio, 2014).

Participantes. La población con la cual se contó en el presente estudio fue de 418 Estudiantes, los cuales se encontraban presentes en las visitas realizadas en los salones de clase para el I Periodo del año 2017. Un total de 82 individuos se consideraron Víctimas del Conflicto; de estos, 47Estudiantes aceptaron participar.

Procedimiento. Inicialmente, se llevó a cabo una revisión de la literatura, acerca de la historia del Conflicto Armado a nivel nacional y local; también sobre las modalidades de violencia en el marco del Conflicto, sobre los niveles de resiliencia, las estrategias de afrontamiento y la salud general de los individuos afectados por hechos violentos.

Luego de esta revisión teórica, se hizo un estudio exploratorio acerca de la población de Estudiantes Víctimas del Conflicto. Se encontró, en las estadísticas de la Universidad, que había pocos Estudiantes del Programa identificados como Víctimas del Conflicto y esa información era confidencial: no se podía contar con ella. Entonces, se produjo la necesidad de ir a cada salón, haciendo la reflexión sobre el Conflicto Armado y sobre la Ley de Víctimas; se preguntó quiénes, a partir de la información suministrada sobre la Ley de Víctimas 1448, se consideraban Víctimas del Conflicto Armado. Una vez identificados los Estudiantes que se consideraban Víctimas, se les suministró el consentimiento informado para que lo firmaran, y, seguidamente, se les aplicó la batería de pruebas.

Instrumentos. Los instrumentos utilizados desde la perspectiva de la presente investigación permitieron recolectar la información necesaria para el trabajo realizado, utilizando, desde los parámetros del enfoque cuantitativo, los siguientes instrumentos:

Encuesta de Datos Sociodemográficos (Ad hoc). La encuesta de datos sociodemográficos es un método de recolección de información aplicada a una persona o población determinada. Asimismo, son utilizadas para la recolección de datos referidos principalmente al componente personal; estas incluyen preguntas relacionadas con el estado civil, edad, género, nivel de escolaridad entre otros (Figueroa \& Trejo, 2003).

Encuesta de Datos sobre la Vivencia del Conflicto Armado (Ad hoc). En esta encuesta se incluyeron preguntas sobre hechos victimizantes, la posibilidad de estudiar Psicología, experiencia en el Conflicto, la atención psicológica recibida, entre otros aspectos; los cuales permitieron conocer la realidad de las victimas detalladamente. 
Escala de Salud General de Goldberg (Golberg \& Blackwell, 1970). Esta escala fue elaborada por Goldberg y Blackwell, en la década de 1970, para el Hospital de Manchester, Inglaterra. Fue de gran apoyo para los médicos y profesionales de la salud, quienes necesitaban conocer y evaluar los niveles de salud general de sus pacientes; por esta razón, tomó el nombre de Cuestionario de Autoinforme de Salud General.

El cuestionario inicial contaba con 60 Ítems, pero, a medida que pasaba el tiempo, se iban diseñando nuevas versiones abreviadas, por lo que actualmente consta de 12 Ítems. El concepto de salud general percibida, hace referencia a la identificación autopercibida por las personas frente a la incapacidad de estas, para ejecutar actividades diarias normales y adaptativas, como también la presencia de fenómenos perturbadores y sintomatología psicopatológica, tal y como lo aborda la escala de salud general elaborada por Goldberg y Blackwell en 1970 (Villa, Zuluaga Arboleda, \& Restrepo Roldán, 2013).

Existen diversos estudios que evidencian las propiedades psicométricas y utilidad de la escala de Goldberg; muestra de ello, es el estudio de tipo instrumental, desarrollado por Rocha et al., 2011, que tuvo como objetivo principal analizar las propiedades psicométricas de la escala de salud general (GHQ-12 por sus siglas en ingles), como un instrumento de cribado en una muestra representativa de la población española, contando a su vez con población mayor de 65 años. Los resultados de esta investigación muestran que cada uno de los ítems del GHQ se correlaciona con los demás, lo que muestra valores de correlación superiores a 0,51 . Por otra parte, el análisis factorial exploratorio confirma que gran parte de la variabilidad del instrumento (67\% Likert y $73 \%$ GHQ) puede ser explicada al reunir todos los ítems en un único factor. El GHQ-12 demuestra una alta consistencia interna en población general y también en población mayor de 65 años.

De igual manera, otro estudio que también resalta el análisis de la estructura factorial de esta escala, y da a conocer la mejor forma de puntuación para el GHQ-12 en la población de Bucaramanga-Colombia, evidencia que, en esta, la forma de puntuación ordinal presenta un mejor comportamiento psicométrico que las binarias. Todas las formas de puntuación muestran alta consistencia interna y aceptable solución factorial, excepto la forma 0-0-0-1 (Campo, 2007).

Escala de Resiliencia de Adultos (Wagnild \& Young, 1993). Esta escala fue diseñada para determinar las cualidades personales que favorezcan la adaptación resiliente de manera individual. Para su construcción, se tuvo en cuenta el relato de 34 mujeres mayores de Estados Unidos, las cuales habían superado de manera positiva las situaciones problemáticas de su vida. La escala cuenta con 25 ítems que van desde 1, que equivale a Totalmente en Desacuerdo, a 5, que es Totalmente de Acuerdo. En esta escala se establecen dos factores: competencia personal y aceptación de sí mismo y de la vida. Además, puede ser administrada de manera colectiva o individual, y sin límite de tiempo (Rodríguez, y otros, 2009).

Análisis Estadístico. Para llevar a cabo el análisis estadístico, se utilizó el paquete estadístico Statiscal Package for Social Science (SPSS, v20). Con la ayuda de este paquete se obtuvieron frecuencias, porcentajes, medias y sus desviaciones típicas.

\section{RESULTADOS}

Datos Sociodemográficos. De acuerdo con las características sociodemográficas de los participantes del estudio, se tiene en cuenta que, en su mayoría, la muestra estaba conformada por Estudiantes del sexo femenino con un 78,7 \%; a su vez, la mayor cantidad de participantes tenían un rango de edad entre 17 y 19 años, representados con el 40, 4 \%; de igual forma, el nivel socioeconómico con más alto porcentaje fue el Medio-Bajo y Medio, con 31,9 \% cada uno. Para ver más detalladamente los resultados ver Tabla 1. 
Búsqueda. 7(24):e465 Enero-Junio, 2020. https://doi.org/10.21892/01239813.465

Tabla 1. Características Sociodemográficas de los Participantes.

\begin{tabular}{lcc}
\hline Características & N & \% \\
\hline Sexo & 37 & 78,7 \\
Femenino & 10 & 21,3 \\
Masculino & & \\
Edad & 19 & 40,4 \\
$17-19$ & 18 & 38,3 \\
$20-24$ & 10 & 21,3 \\
$25-32$ & & \\
Nivel Socioeconómico & 13 & 27,7 \\
Bajo & 15 & 31,9 \\
Medio-Bajo & 4 & 8,5 \\
Medio-Alto & 13 & 27,7 \\
Alto & 2 & 4,2 \\
Perdidos & & \\
\hline
\end{tabular}

Aspectos Relacionados con Hechos Victimizantes y con la Atención Psicosocial. El tipo de hecho victimizante más frecuente fue el Desplazamiento Forzado (44,7 \%), seguido del Asesinato $(21,3 \%)$ y las amenazas (6.4\%). Esta información se encuentra más detallada en la Tabla 2.

Tabla 2. Número y Tipos de Hechos Victimizantes.

\begin{tabular}{lcc}
\hline Número de Hechos & $\mathbf{N}$ & \% \\
\hline 1 & 37 & 78,8 \\
2 & 9 & 19,1 \\
3 & 1 & 2,1 \\
Tipo de Hecho & 21 & 44,7 \\
Desplazamiento & 10 & 21,3 \\
Asesinato & 3 & 6,4 \\
Amenazas & 1 & 2,1 \\
Atentado & 2 & 4,2 \\
Secuestro & 2 & 4,2 \\
Testigo y Desplazado & 2 & 4,2 \\
Tortura y Asesinato & 2 & 4,2 \\
Asesinato y Desplazamiento & 1 & 2,1 \\
Secuestro y Desplazamiento & 1 & 2,1 \\
Desplazamiento y Abuso & 1 & 2,1 \\
Desplazamiento y Masacre & 1 & 2,1 \\
Desplazamiento, Asesinato y Reclutamiento & 2 \\
\hline
\end{tabular}

El 44,7\% de los participantes consideraron que los hechos vividos en el Conflicto influyeron para que estudiaran Psicología. Aunque el 34\% consideraba que necesitaba apoyo psicosocial, solo el $17 \%$ ha recibido ayuda psicológica. El $76 \%$ estimaría conveniente recibir apoyo psicosocial por parte de Bienestar Universitario. Esta información se encuentra detallada en la Tabla 3. 
Anaya-Mercado et al - Niveles de Resiliencia y Estado de Salud en Estudiantes de Psicología, Víctimas del Conflicto Armado

Tabla 3. Aspectos Relacionados con Hechos Victimizantes y Atención Psicosocial.

\begin{tabular}{lcc}
\hline \multicolumn{1}{c}{ Pregunta } & $\mathbf{N}$ & $\%$ \\
\hline Las vivencias traumáticas influyen en su proceso de aprendizaje. & 17 & 36,2 \\
Los hechos traumáticos vividos influyeron para que estudiara Psicología & 21 & 44,7 \\
Las vivencias traumáticas influyeron en su vida familiar & 24 & 51,1 \\
Recibió atención psicosocial por los hechos victimizantes & 8 & 17 \\
Fue significativa la ayuda recibida a nivel psicosocial & 11 & 23,4 \\
La Universidad le ha brindado atención psicosocial & 9 & 19,1 \\
Necesita apoyo psicosocial & 16 & 34,0 \\
Asistiría a consulta psicológica en Bienestar & 36 & 76,6 \\
\hline
\end{tabular}

Niveles de Resiliencia en los Participantes y Salud General percibida. Los niveles de resiliencia en los participantes del estudio varían de Escasa Resiliencia a Alta Resiliencia. La mayoría de los Estudiantes presentan Resiliencia Alta y Moderada; sólo 8,5\% parece tener una Escasa Resiliencia frente a los eventos traumáticos vividos en el Conflicto. Esta información se encuentra detallada en la Tabla 4.

Tabla 4. Niveles de Resiliencia en los Estudiantes.

\begin{tabular}{ccc}
\hline & n* & \% \\
\hline Escasa Resiliencia & 4 & 8,5 \\
Resiliencia Moderada & 21 & 44,7 \\
Alta Resiliencia & 22 & 46,8 \\
Salud General Percibida & M* & DE* \\
\hline
\end{tabular}

*: $\mathrm{n}=$ frecuencia; $M=$ media; $\mathrm{DE}=$ desviación estándar

\section{DISCUSIÓN}

El objetivo principal de esta investigación fue determinar los niveles de resiliencia, el estado de salud general percibido y los aspectos relacionados con los hechos victimizantes y con la atención psicosocial de Estudiantes Universitarios Víctimas del Conflicto en Sucre. Los Estudiantes de la muestra fueron víctimas de múltiples hechos victimizantes, tales como el Desplazamiento, el Asesinato, las amenazas, atentados, secuestro, entre otras.

El hecho victimizante más frecuente es el Desplazamiento, situación que, según cifras de la Red Nacional de Información (RNI), siempre estará en primer lugar, dado que para el 01 de abril del año 2019, se reportaron 7.478 .723 personas como desplazadas. El segundo hecho victimizante, fue el Asesinato u Homicidio, información que también es demostrada en la RNI, puesto que, para esta misma fecha, 880.039 individuos declararon haber tenido familiares cercanos víctimas de Asesinato (Red Nacional de Información, 2019). 
Asimismo, el informe realizado por el Centro de Memoria Histórica, que documentó hechos sucedidos en el Conflicto Armado entre 1958 y julio de 2018, señala que el Conflicto Armado colombiano dejó como resultado 262.197 muertos; de este gran número de víctimas, 215.005 eran civiles y 46.813 eran combatientes. Este número de personas que quedaron sin vida fueron víctimas de modalidades de violencia utilizada por los actores del Conflicto Armado, específicamente estos realizaron asesinatos selectivos, masacres, atentados terroristas, desapariciones forzadas, secuestros, violaciones, y utilización de minas. De igual forma, el Observatorio de Memoria y Conflicto informa que el Conflicto colombiano ha dejado 80.514 desaparecidos, 37.094 víctimas de secuestro, 15.687 víctimas de violencia sexual y 17.804 menores de 18 años reclutados (Centro Nacional de Memoria Histórica, 2018).

Por otro lado, es importante considerar que todos estos hechos han sido traumáticos para la vida de los Estudiantes, principalmente en su entorno familiar, pues, al realizar la prueba, se obtuvo que el $51,1 \%$ presentaba una afectación en su vida de familia a partir de lo vivido. Según Guerrero (2011), los sucesos violentos relacionados con el Conflicto Armado Interno, afectan de manera directa los derechos de muchas familias, obligándolas a dejar sus hogares, territorios de origen y desplazarse a otras ciudades, aumentando, así, las cifras de personas que viven en la pobreza extrema y padeciendo de necesidades básicas insatisfechas, como son tener una vivienda, educación, estar afiliados a una IPS y contar con un trabajo estable, lo cual causa daños en la integridad física y moral de estas víctimas trayendo como resultado una afectación mucho más grave del núcleo familiar (Guerrero, 2011).

Teniendo en cuenta lo anterior, es imperante para los Estudiantes recibir ayuda psicológica, dado que la atención psicosocial a víctimas, busca contribuir con la reparación o mitigación de los daños generados por el Conflicto Armado, como lo es el impacto a la integridad psicológica y moral, proyecto de vida abatido y sufrimiento emocional. Esta atención aporta al restablecimiento de los lazos familiares, tejido social y cohesión socio cultural. Las acciones con relación a esto están direccionadas a mejorar la calidad de vida de las víctimas, tratar las afecciones mentales y disminuir la permanencia de consecuencias provenientes de los sucesos traumáticos vividos (Ministerio de Salud, 2016).

No obstante, dentro de esta investigación se pudo constatar que solo el $17 \%$ ha recibido apoyo psicológico, lo cual demuestra un nivel Muy Bajo de Estudiantes con un soporte terapéutico formal, aunque el 34\% de estos reconocen que necesitan de esta atención. Situación que también se evidenció en un estudio realizado en 2015, con adultos expuestos al Conflicto Armado, ya que encontraron que esta población tenía una baja percepción en la atención de la salud, con un total de $1.64 \%$, pero con una alta necesidad de la misma, pues arrojaron un porcentaje de $2.35 \%$, es decir, estas personas reconocían la necesidad de la atención psicológica, pero no asistían a estas (Hewitt \& cols., 2016).

Es necesario recalcar que, aunque los Estudiantes que participaron en la presente investigación no demostraron que asistieron a algún tratamiento psicológico, se encontró que el $46.8 \%$ de ellos han tenido un alto nivel de resiliencia ante todos esos difíciles sucesos por los que han pasado. De igual forma, el $44.7 \%$ de ellos ha señalado que han tenido un nivel Moderado de resiliencia, de lo cual se puede inferir que estas personas, frente a estos hechos, han sido capaces de afrontar el dolor, la angustia y el trauma.

De igual manera, se relacionan estos resultados con las diferentes redes de apoyo con las que probablemente contaron los Estudiantes Víctimas del Conflicto, debido a que, tal como lo menciona Ibáñez, citado por Sánchez (2015), las experiencias negativas nos pueden ayudar a ser mejores personas, en cualquiera de los roles que representemos (padres, hermanos, profesionales) y, en general, nos convierte en mejores ciudadanos; pues, al transcurrir el tiempo, esta capacidad se va desarrollando, brindándole la oportunidad a las personas de adaptarse mejor a las situaciones adversas.

Por ello, es importante la participación de las redes de apoyo en el proceso de resiliencia de las personas; una de las primeras funciones que se destacan de estas redes, es el aporte como compañía y apoyo, entre las cuales estarían las redes de parentesco y las redes de amistad. 
Otras redes que funcionan son las que aportan una guía cognitiva, las cuales facilitan el acceso a la información y al conocimiento respecto a algún tema de interés (redes académicas, redes sociales profesionales). Finalmente, destacamos el acompañamiento que cumplen aquellas redes en función del servicio a la comunidad (Sánchez Salazar, 2015).

Otro hecho que pone de manifiesto la capacidad de resiliencia que despliegan las personas, frente a hechos violentos, ha sido la investigación realizada en 2015 con personas adultas expuestas al Conflicto Armado, en donde se halló que $61 \%$ de la población tuvo un nivel moderado de resiliencia, y 32\%, un nivel alto de resiliencia (Hewitt \& cols., 2016). Los autores indican que, a pesar de que las cifras varían, en comparación con los resultados del presente estudio, las personas que han atravesado estos eventos, han realizado lo posible por desarrollar la capacidad de resiliencia, logrando sobrevivir y dándose una nueva oportunidad de desarrollo y progreso.

No obstante, para que todas estas personas puedan tener un equilibro emocional, mental y social, es de gran importancia que puedan asistir a terapias psicológicas. En Colombia, las Víctimas del Conflicto Armado tienen acceso a una atención integral en salud. El modelo MIAS (Modelo Integral de Atención en Salud) tiene como meta principal colocar el sistema de salud hacia una dirección en la cual se brinden condiciones óptimas para la atención de esta población, bajo medidas de equidad y priorización, con una visión centrada en el bienestar de la salud de las personas (Ministerio de Salud, 2016). No obstante, vale la pena preguntarse si los Estudiantes Universitarios algún día han hecho uso de este modelo o si saben del derecho que tienen a la atención integral en salud.

Con respecto a los resultados obtenidos en la aplicación del Cuestionario de Salud General de Goldberg GHQ-12, es interesante comparar las medias obtenidas con otros estudios que hayan usado la misma escala. En nuestra investigación, se evidencia una media de 6,81 y una desviación estándar de: 1,38; esto contrasta con el 5.34 (2.77) encontrado en la ciudad de Medellín (Colombia). La diferencia puede deberse a que la muestra del segundo estudio fue hospitalaria, por eso, la percepción general de salud fue menor (Villa, Zuluaga y Restrepo, 2013). No encontramos ningún estudio sobre salud general percibida en jóvenes víctimas del conflicto armado, por esta razón no podemos comparar los resultados con otros estudios.

Por último, se recomienda, para futuras investigaciones, ampliar la búsqueda sobre este tema no solo en Estudiantes de Psicología de una Universidad, sino también extenderlo a Universidades de la región que oferten esta carrera, debido a que es relevante conocer el nivel de resiliencia y el estado de salud mental percibido a nivel regional y no solo local, tomando en cuenta los pocos estudios hallados en relación a esta problemática en la región.

\section{CONCLUSIONES}

Se destaca en esta investigación, que los Estudiantes Universitarios de Psicología, que han sido Víctimas del Conflicto Armado en Sucre, experimentaron hechos victimizante que marcaron sus vidas de manera significativa, tales como el Desplazamiento y el Homicidio, realizados por grupos armados ilegales; situación que también afectó sus áreas familiares, sociales y personales. Además, se halló que posiblemente estos hechos influyeron en la elección de la carrera profesional de Psicología. Sin embargo, estos Estudiantes han demostrado que, aunque han sido resilientes a la situación, no contaron con un apoyo psicológico especializado, lo cual actualmente reconocen como necesario para poder afrontar todo lo que vivieron.

Se hace preciso que los Estudiantes Víctimas del Conflicto reciban una atención psicológica de calidad, y se resalta la importancia de un tratamiento adecuado para estos jóvenes. Además, se destaca el papel de la Universidad en la mejora del proceso de resiliencia de estos jóvenes.

\section{Conflicto de intereses}

Los autores declaran no tener ningún conflicto de intereses. 


\section{REFERENCIAS}

Bonilla, A. (2013). Universidad del Valle sede Pacifico. Obtenido de http://bibliotecadigital. univalle.edu.co/bitstream/10893/10624/1/CB-0503495.pdf

Castaño, G., Sierra, G., Sánchez, D., Semenova, N., Salas, C., Buitrago, C., \& Agudelo, M. (2018). Universidad CES. Obtenido de http://bdigital.ces.edu.co:8080/repositorio/ handle/10946/4722

Castillo, S., Romero-Acosta, K., Sahagún-Navarro, M., \& Sánchez-Vergara, A. (2017). Evaluación de la salud mental de personas mayores, Víctimas del Conflicto Armado, atendidas en el Centro de Orientación Sociojurídica de Sincelejo, en los años 2012 - 2013. Revista Búsqueda, $18,91-100$.

Campo-Arias, A. (2007). Cuestionario general de salud-12: análisis de factores en población general de Bucaramanga, Colombia. Revista Iatreia, 20 (1).

Centro Nacional de Memoria Histórica. (2018). Centro Nacional de memoria histórica. Obtenido de http://www.centrodememoriahistorica.gov.co/micrositios/un-viaje-por-la-memoriahistorica/pdf/cifras.pdf

Centro Nacional de Memoria Histórica. (2018). Centro Nacional de Memoria Histórica. Obtenido de http://www.centrodememoriahistorica.gov.co/noticias/noticias-cmh/262-197-muertosdejo-el-Conflicto-armado.

Congreso de Colombia. (2011). Unidad para la Atención y la Reparación Integral a las Víctimas. Obtenido de https://www.unidadvictimas.gov.co/es/Ley-1448-de-2011/13653

Figueroa, D., \& Trejo, E. (2003). Academia. Obtenido de http://www.academia.edu/4272784/ Las_encuestas_sociodemograficas

Golberg, D., \& Blackwell, B. (23 de May de 1970). Psychiatric illness in general practice: a detailed study using a new method of case identification. $\mathrm{Br}$ Med J, 2, 439.

Guerrero, M. (2011). Afectación de la familia a causa del Conflicto Armado interno. Estudiositas. Grupo de Investigación Política Pública y Facultad de Derecho de la Universidad Católica de Colombia.

Herrera, E. (2016). Universidad cooperativa de Colombia. Obtenido de https://www.ucc.edu. $\mathrm{co} / \mathrm{prensa/2016/Paginas/consecuencias-en-la-salud-mental-de-actores-y-victimas-del-}$ Conflicto-armado.aspx

Hewitt Ramírez, N., Juárez, F., Parada Baños, A., Guerrero Luzardo, J., Romero Chávez, Y., Salgado Castilla, A., \& Vargas Amaya, M. (2016). Afectaciones psicológicas, estrategias de afrontamiento y niveles de resiliencia de adultos expuestos al Conflicto Armado en Colombia. Revista Colombiana de Psicología, 25(1), 125-140.

Médicos Sin Fronteras . (2013). Asociación Colombiana de Psiquiatria. Obtenido de http:// psiquiatria.org.co/web/wp-content/uploads/2013/06/Las-Heridas-Menos-Visibles-ReporteMSF-2013.pdf

Ministerio de Salud. (2016). MINSALUD. Obtenido de http://www.iets.org.co/victimas/Documents/ Documento_alcance_objetivos_indicaciones_consenso_24\%20nov.pdf 
Anaya-Mercado et al - Niveles de Resiliencia y Estado de Salud en Estudiantes de Psicología, Víctimas del Conflicto Armado

Ministerio de Salud. (2016). MINSALUD. Obtenido de http://www.saludcapital.gov.co/SU_ Espacio_Vivienda/Fichas_tecnicas/PAPSIVI/Orientaciones_Individuales.pdf

Muñoz, A. (2019). About Español. Obtenido de https://www.aboutespanol.com/que-es-laresiliencia-2396440

Ramírez-Giraldo, A., Hernández-Bustamante, O., Romero-Acosta, K., \& Porras-Mendoza, E. (2017). Trastornos de ansiedad y del estado de ánimo en personas Víctimas del Conflicto Armado en Colombia: el caso de Chengue y de Libertad. Psicología desde el Caribe, 34(1), $30-41$.

Red Nacional de Información. (2018). Unidad de victimas. Obtenido de https://cifras. unidadvictimas.gov.co/Home/Victimizaciones

Red Nacional de Información. (2019). Unidad de victimas. Obtenido de https://cifras. unidadvictimas.gov.co/Home/Victimizaciones

Rocha, K., Perez. K., Rodríguez-Sanz. M., Borrell. C., Obiols. J. (2011). Propiedades psicométricas y valores normativos del General Health Questionnaire (GHQ-12) en población general española. International Journal of Clinical and Health Psychology, 11 (1), 125-139.

Rodríguez, J., De La Torre, A., \& Miranda, C. (2002). Biomédica. Obtenido de file:///C:/Users/ usuario/Downloads/1183Texto\%20del\%20manuscrito\%20completo\%20(cuadros\%20y\%20 figuras\%20insertos)-4804-1-10-20120923.pdf

Rodríguez, M., Pereyra, M., Gil, E., Jofré, M., De Bortoli, M., \& Labiano, L. (2009). Propiedades psicométricas de la escala de resiliencia versión argentina. Evaluar, 9, 72 - 82.

Sampieri, R., Collado, C., \& Lucio, P. (2014). Metodología de la Investigación (sexta ed.). Mexico D.F: Mc Graw Hill Education.

Sánchez Salazar, M. (2015). Universidad Cuauhtemoc. Obtenido de http://servicios.ucuauhtemoc. edu.mx/biblioteca/tesis/tesis_mcepc/REDES\%20SOCIALES\%20DE\%20APOYO\%20 COMO $\% 2$ 20FACTOR $\% 20$ DE $\% 20$ MEJORAMIENTO $\% 20$ EN $\% 20$ LA $\% 20$ CONDUCTA $\% 20$ RESILIENTE\%20EN\%20ADOLESCENTES.pdf

Solis-Cámara, P., Meda-Lara, R., Moreno-Jiménez, B., Juárez-Rodríguez, P. (2016). Estructura factorial del cuestionario de salud general ghq-12 en población general de méxico. Revista Salud \& Sociedad de la Universidad Católica del Norte, 7 (1), 62-76.

Villa, I., Zuluaga Arboleda, C., \& Restrepo Roldán, L. (2013). Propiedades psicométricas del Cuestionario de Salud General de Goldberg GHQ-12 en una institución hospitalaria de la ciudad de Medellín. Avances en Psicología Latinoamericana, 31 (3), 532-545.

Wagnild, G., \& Young, H. (1993). Development and Psychometric Evaluaction of the Resilience Scale. Journal of Nursing Measurment, 1(2), 165 - 178. 